\title{
Gauging the twisted Poincaré symmetry as noncommutative theory of gravitation
}

\author{
M. Chaichian ${ }^{a}$, M. Oksanen ${ }^{a}$, A. Tureanu ${ }^{a}$ and G. Zet ${ }^{b}$ \\ ${ }^{a}$ Department of Physics, University of Helsinki \\ and Helsinki Institute of Physics, P.O. Box 64, FIN-00014 Helsinki, Finland \\ ${ }^{b}$ Department of Physics, "Gh. Asachi" Technical University, \\ Bd. D. Mangeron 67, 700050 Iasi, Romania
}

\begin{abstract}
Einstein's Theory of General Relativity was formulated as a gauge theory of Lorentz symmetry by Utiyama in 1956, while the Einstein-Cartan gravitational theory was formulated by Kibble in 1961 as the gauge theory of Poincaré transformations. In a noncommutative space-time with canonical commutation relations between the coordintes, Lorentz symmetry is violated and field theories constructed on such space-times have instead the so-called twisted Poincaré invariance. In this paper a gauge theory formulation of noncommutative gravity is proposed based on the twisted Poincaré symmetry together with the requirement of covariance under the general coordinate transformations, an essential ingredient of the theory of general relativity. The advantages of such a formulation as well as the related problems are discussed and possible ways out are outlined.
\end{abstract}

\section{Introduction}

It is generally expected that the smooth manifold structure of the classical space-time should break down at distances of the order of the Planck length,

$$
l_{\mathrm{P}}=\sqrt{\frac{\hbar G}{c^{3}}} \approx 1.6 \cdot 10^{-35} \mathrm{~m},
$$

so that all physical phenomena become essentially nonlocal - as opposed to the locality of traditional geometrical theories of gravitation and quantum and gauge field theories of particle physics. It is hoped that an appropriate implementation of the nonlocality will eventually enable the formulation of a unified theory of the fundamental interactions of Nature, which should be free from singularities, divergences and any other kind of inconsistences. The noncommutativity of space-time coordinates is one way to implement the nonlocality of Planck scale physics, which is well motivated. 
Formally, the noncommutativity of coordinate operators $x^{\mu}, \mu=0,1,2,3$, is achieved by imposing the commutation relations

$$
\left[\hat{x}^{\mu}, \hat{x}^{\nu}\right]=i \theta^{\mu \nu}
$$

where in the canonical case $\theta^{\mu \nu}$ is an antisymmetric constant matrix of dimension lengthsquared, and by letting the fields on noncommutative space-time be functions of the noncommutative coordinate operators. Through Weyl quantization the noncommutative algebra of operators generated by (1.2) can be represented on the algebra of ordinary functions on classical space-time by using the noncommutative Moyal $\star$-product. The more general case with $\theta^{\mu \nu}$ being an antisymmetric tensor field has also been considered.

The idea that space-time coordinates do not commute can be seen as a generalization of the corresponding property of the quantum mechanical phase space of coordinate $\hat{x}^{i}$ and momentum $\hat{p}_{j}$ operators,

$$
\left[\hat{x}^{i}, \hat{p}_{j}\right]=i \hbar \delta_{j}^{i}
$$

The first "quantized space-time", which was based on a noncommutative algebra of coordinate operators, was introduced in [1].

Combining Einstein's theory of general relativity and quantum mechanical measurements obeying Heisenberg's uncertainty principle leads to operational noncommutativity of space-time coordinates [2, 3]. It is not possible to measure distances of the order of the Planck length, because it would require so much energy to be present in the localization region of a measurement, that the gravitational interaction would prevent any signal from leaving the region, i.e. the localization of the measurement would collapse under its own gravity. This has led to the formulation of QFT on noncommutative space-time.

String theory is one of the strongest motivations for considering noncommutative spacetime geometries and noncommutative gravitation. It has been shown that when the end points of strings in a theory of open strings are constrained to move on $D$-branes in a constant (supergravity) $B$-field background and the theory is taken in a certain low-energy limit, then the full dynamics of the theory is described by a gauge theory on a noncommutative spacetime [4]. In this Seiberg-Witten (low-energy) limit [4], the open string modes completely decouple from the closed string modes and only the end point degrees of freedom for the open strings are left to live on a noncommutative space-time defined by the coordinate commutation relations (1.2). Thus noncommutative gauge theory emerges as a low-energy limit of open string theory with constant antisymmetric background field.

The formulation of local (gauge) symmetries on a noncommutative (nonlocal) space-time is a delicate issue. Most gauge groups can not be defined on noncommutative space-time, because they do not close under the $\star$-product. The noncommutative unitary group $U_{\star}(n)$ can be defined. Its representations, however, are limited by the no-go theorem [5] (see also [6, 7]) stating that only the fundamental, anti-fundamental and adjoint representations of the gauge group $U_{\star}(n)$ are allowed and matter fields can be charged under at most two 
noncommutative simple gauge groups. A noncommutative Standard Model based on the gauge groups $U_{\star}(n)$ has been constructed [8] (see also, for its extension to noncommutative MSSM, 9]).

Another approach to the noncommutative gauge theories has been through the so-called Seiberg-Witten map [4], which originally related a noncommutative $U_{\star}(n)$ gauge theory to a commutative one, obtained as low-energy effective limits in string theory, by using two different regularization methods (the point-splitting method and the Pauli-Villars method, respectively). The philosophy behind the Seiberg-Witten map has been subsequently used to extend the possible noncommutative gauge groups to include special unitary groups as well: indeed, noncommutative gauge theories with gauge fields valued in the enveloping algebra of $s u(n)$ have been constructed [10, 11] and a corresponding noncommutative version of the Standard Model has been built [12].

A new interpretation of the relativistic invariance of the commutation relations (1.2) was proposed in [13]: by using the concept of twisted Poincaré algebra, the relativistic invariance can be generalized to the framework of Hopf algebras. If in the usual (commutative) case, relativistic invariance means invariance under the Poincaré transformation, then in the noncommutative case relativistic invariance means invariance under twisted Poincaré transformations [14]. Since the twist deformation of the Poincaré algebra does not affect the multiplication of the algebra generators, the structure of the algebra is preserved, and consequently the representation content of the twisted Poincaré algebra is identical with that of the usual Poincaré algebra. This legitimates the usage of the familiar representations of the Poincaré symmetry in the context of noncommutative field theories [13]. The noncommutative field theories, although they lack the Lorentz symmetry, are invariant under the twisted Poincaré algebra, deformed by the Abelian twist element

$$
\mathcal{F}=e^{\frac{i}{2} \theta^{\mu \nu} P_{\mu} \otimes P_{\nu}}
$$

where $P_{\mu}=-i \partial_{\mu}$ are the generators of space-time translations. The twist induces, on the representations of the Poincaré algebra, the deformed multiplication

$$
\mu(\phi \otimes \psi)=\phi \psi \longrightarrow \mu_{\star}(\phi \otimes \psi)=\mu\left(\mathcal{F}^{-1}(\phi \otimes \psi)\right)=\phi \star \psi,
$$

which is precisely the (Moyal) «-product. The question about the action of the twisted Poincaré algebra on fields and of the actual meaning of the invariance under twisted Poincaré algebra has been raised in [15], where it was proposed to seek the answer by re-constructing the fields using the method of induced representations, in a manner compatible with the twisted Poincaré algebra. Along these lines of thought, a new interpretation of the noncommutative fields has been proposed in [16], according to which the noncommutative fields carry representations of the full Lorentz group, but admit transformations only under the residual symmetry which preserves the matrix $\theta_{\mu \nu}$ invariant.

Recently, an attempt was made to twist also the gauge algebra, by extending the global Poincaré algebra through a semidirect product with the gauge algebra, and by twisting the 
coproduct of the combined algebra by using the Abelian twist element (1.4). This approach was shown [17] to be in conflict with the very idea of gauge symmetry, since it implicitly assumed that when a field transforms according to a given representation, then its partial derivatives of any order also transform in the same representation of the gauge algebra, which is obviously not the case.

The question arises whether the concept of twist provides a symmetry principle for formulating noncommutative field theories: meaning that any symmetry such theories may enjoy, be it space-time or internal, global or local, should be formulated as a twisted symmetry. It has been shown [18] that it is not possible to twist the internal gauge transformations and at the same time keep the Moyal space-time structure defined by (1.2). It is intriguing that the external Poincaré symmetry and the internal gauge symmetry can not be unified under a common twist. This situation is reminiscent of the Coleman-Mandula theorem [19], although not entirely, since this theorem concerns global symmetry and simple groups. However one can envisage that supersymmetry [20], due to its intrinsic internal symmetry, may reverse the situation, and a noncommutative gauge theory may be constructed by means of a twist.

There is a good understanding of the noncommutative effects on matter and gauge fields defined on the flat noncommutative space-time. The next step is to incorporate gravity by considering curved noncommutative space-times. The main problem is that the noncommutativity parameter $\theta^{\mu \nu}$ is usually taken to be constant, which breaks the Lorentz invariance of the commutation relations (1.2), and implicitly of any noncommutative field theory. This has motivated a large amount of work to study noncommutative deformations of general relativity (see, e.g., [21]-[?] and references therein). Noncommutative gauge theory defined through matrix models [27, 28] contains a specific version of gravity as an intrinsic part, and provides a dynamical theory on noncommutative spaces. Noncommutative deformations of gravity have also led to a complex metric and gauge groups larger than the Lorentz group [21, 23]. A noncommutative General Relativity restricted to the volume-preserving transformations (unimodular theory of gravity) has been also constructed [29]. Lately, the version of noncommutative gravity obtained by the deformation of the diffeomorphism algebra [24] using the twist introduced in [13] has been most studied in the literature. However, it turned out that the dynamics of the noncommutative gravity arising from string theory [30] is much richer than this version of noncommutative gravity. The dynamics of closed strings in the presence of a constant $B$-field induces a gravitational action in the next-to-leading order in the Seiberg-Witten limit [4]. Some of the three-graviton vertices have been derived and they can not be obtained from an action written only in terms of the $\star$-product. It is suspected that the reason for this is the non-invariance of the Moyal $\star$-product under space-time diffemorphisms. A geometrical approach to noncommutative gravity, leading to a general theory of noncommutative Riemann surfaces in which the problem of the frame-dependence of the $\star$-product is also recognized, has been proposed in [31].

A possibility to obtain a theory which is covariantly deformed under the local Poincaré 
transformations is that of gauging the twisted Poincaré algebra itself. Einstein's Theory of General Relativity was formulated as a gauge theory of Lorentz symmetry by Utiyama 32 in 1956, while the Einstein-Cartan gravitational theory was formulated by Kibble [33] in 1961, as the gauge theory of Poincare transformations. Instead of the partial derivatives in the Abelian twist element (1.4) one can use the covariant derivatives [33] (see also [34]):

$$
\nabla_{\mu}=\partial_{\mu}+\frac{i}{2} \omega_{\mu}^{a b} \Sigma_{a b}
$$

where the (constant) matrices $\Sigma_{a b}$ form a representation of the Lorentz algebra. We can define a covariant non-Abelian twist element as

$$
\mathcal{T}=e^{-\frac{i}{2} \theta^{\mu \nu} \nabla_{\mu} \otimes \nabla_{\nu}+\mathcal{O}\left(\theta^{2}\right)}
$$

with possible covariant higher order terms in the noncommutativity parameter $\theta^{\mu \nu}$ in the exponent. In this paper we study the properties of such a covariant twist.

In Section 2 we review the commutative gauge theory of gravitation. The gauge covariant derivative is defined by considering the Poincaré algebra as gauge symmetry. The curvature and torsion tensors are obtained from the commutator of covariant derivatives. These results show that the Poincaré gauge theory of gravitation has the structure of a Riemann-Cartan space $\mathcal{U}_{4}$ with both curvature and torsion.

In Section 3, the mathematical framework of twisted Hopf algebras is explained and the twisted Poincaré algebra is defined. The concept of relativistic invariance in the noncommutative theory is understood as invariance under the twisted Poincaré transformations.

Section 4 is devoted to the possibility of gauging the twisted Poincaré symmetry itself. A covariant non-Abelian twist element is defined by using the covariant derivative of the Poincaré gauge theory. The conditions ensuring that the Hopf algebra structure is preserved by the twist are verified. It is shown that the $\star$-product induced by the covariant twist is not associative. Therefore, the twisted Poincaré symmetry can not be gauged by generalizing the Abelian twist (1.4) to a covariant non-Abelian twist (1.7), nor by introducing a more general covariant twist element.

\section{Commutative gauge theory of gravitation}

General Relativity (GR) still lacks the status of fundamental microscopic theory, because of the standing problems of quantization of the gravitational field and the existence of singular solutions under very general assumptions. Since the concept of gauge symmetry has been highly successful in describing the other three fundamental interactions, gauge theories of gravitation are very attractive. The important role of the Poincaré symmetry as the concept of relativistic invariance in the quantum field theory, leads one to consider the Poincaré gauge symmetry as a natural framework for describing the gravitational interaction. 
In the absence of the gravitational field, the underlying space-time symmetry is traditionally described by the global Poincaré group. If we want to obtain a physical theory that is invariant under local Poincaré transformations, the parameters of the transformations will depend on space-time coordinates, and thus new compensating or gauge fields have to be introduced. These fields describe the gravitational interaction and one hopes to obtain a quantum theory in analogy with the internal gauge theories. An important point to be emphasized is that the Poincaré gauge theory of gravitation contains GR as a special case. Its geometric interpretation shows that the space-time has the structure of Riemann-Cartan geometry, possessing both curvature and torsion [33]-37].

The Einstein-Cartan theory of gravitation is a modification of GR, allowing space-time to have torsion, in addition to curvature, and relating torsion to the density of intrinsic angular momentum (the spin). In GR the Lorentz group, instead of the Poincaré group, is the structure group acting on the orthonormal Lorentz frames in the tangent spaces of the space-time manifold. Therefore, there is no room for translations in GR and thus for the torsion and spin tensors. In the Poincaré gauge theory, the torsion and its relation to the spin are naturally introduced, restoring the role of the Poincaré symmetry in relativistic gravity. The curvature and torsion are surface densities of Lorentz transformations and translations, respectively.

The Einstein-Cartan theory is a viable theory of gravitation that differs slightly from Eintein's GR. The effects of spin and torsion can be significant only when the density of matter is very high, but nevertheless much smaller than the Planck density at which quantum gravitational effects are believed to dominate. It is possible that the Einstein-Cartan theory will prove to be a better classical limit for a future quantum theory of gravitation than the theory without spin.

The (global) Poincaré group is a 10-dimensional noncompact Lie group which has the structure of a semidirect product of the translation group $\mathcal{T}_{4}$ and of the Lorentz group $S O(1,3)$

$$
\mathcal{P}=S O(1,3) \ltimes \mathcal{T}_{4}
$$

In order to define its transformations, we consider the Minkowski space-time $\mathcal{M}_{4}$, endowed with the real coordinates $x^{\mu}, \mu=0,1,2,3$. On $\mathcal{M}_{4}$ it is possible to choose global inertial coordinates, such that the infinitesimal interval has the form $\mathrm{d} s^{2}=\eta_{\mu \nu} \mathrm{d} x^{\mu} \mathrm{d} x^{\nu}$, where $\eta_{\mu \nu}=$ $\operatorname{diag}(-1,1,1,1)$ is the Minkowski metric. The isometry group of $\mathcal{M}_{4}$ is the group of global Poincaré transformations, written in the infinitesimal form as

$$
x^{\prime \mu}=x^{\mu}+\omega^{\mu}{ }_{\nu} x^{\nu}+\epsilon^{\mu}
$$

where $\omega^{\mu \nu}=-\omega^{\nu \mu}$ and $\epsilon^{\mu}$ are the ten infinitesimal parameters associated to the Lorentz rotations and space-time translations, respectively.

In order to define matter fields on space-time (scalars, vectors, spinors etc.), we consider the tangent space $T_{p}$ at each point $p \in \mathcal{M}_{4}$. For $\mathcal{M}_{4}$ every $T_{p}$ actually coincides with the 
manifold $\mathcal{M}_{4}$ itself (i.e. $\mathcal{M}_{4}$ is flat and invariable). On each tangent space $T_{p}$ we can use a coordinate frame $(\mathrm{C})$, consisting of four vectors $e_{\mu}$ tangent to the coordinate lines, or a local Lorentz frame $(\mathrm{L})$ of four orthonormal vectors $e_{a}(x)$,

$$
e_{a}(x) \cdot e_{b}(x)=\eta_{a b}=\operatorname{diag}(-1,1,1,1)
$$

which are named the tetrad. The Latin indices $(a, b, \ldots)$ refer to the L-frames and the Greek indices refer to the C-frames. To each L-frame $\left\{e_{a}\right\}$ we can associate local inertial coordinates $x^{a}, a=0,1,2,3$. If the coordinates are globally inertial, one can always choose the tetrad to coincide with the C-frame, $e_{a}=\delta_{a}^{\mu} e_{\mu}$.

A matter field $\phi(x)$ on space-time is always given in the L-frame. In general it is a multi-component object which can be written as a vector-column. The action of a global Poincaré transformation in $T_{p}$ transforms each L-frame into another L-frame, inducing an appropriate (infinitesimal) transformation of the field $\phi(x)$

$$
\begin{aligned}
x^{\prime a} & =x^{a}+\omega^{a}{ }_{b} x^{b}+\epsilon^{a}, \\
\phi^{\prime}\left(x^{\prime}\right) & =\left(1-\frac{i}{2} \omega^{a b} \Sigma_{a b}\right) \phi(x),
\end{aligned}
$$

where $\Sigma_{a b}$ are the spin-matrices which act on the field $\phi(x)$ through matrix-multiplication. For example, if $\phi(x)$ is a spin- $\frac{1}{2}$ Dirac field, then

$$
\Sigma_{a b}=\frac{i}{4}\left[\gamma_{a}, \gamma_{b}\right]
$$

where $\gamma_{a}(a=0,1,2,3)$ are the Dirac matrices. If $\phi(x)$ is a spin- 1 field, then

$$
\left(\Sigma_{a b}\right)_{d}^{c}=i\left(\delta_{a}^{c} \eta_{b d}-\delta_{b}^{c} \eta_{a d}\right)
$$

Equivalently we can write $(\underline{2.2})$ as

$$
\delta \phi(x) \equiv \phi^{\prime}(x)-\phi(x)=-i\left(\frac{1}{2} \omega^{a b} M_{a b}+\epsilon^{a} P_{a}\right) \phi(x)
$$

where

$$
\begin{aligned}
M_{a b} & =i\left(x_{a} \partial_{b}-x_{b} \partial_{a}\right)+\Sigma_{a b} \equiv L_{a b}+\Sigma_{a b} \\
P_{a} & =-i \partial_{a}
\end{aligned}
$$

are the generators of the global Poincaré transformations in the space of fields. These generators satisfy the Poincaré algebra $\mathcal{P}$ :

$$
\begin{aligned}
{\left[P_{a}, P_{b}\right] } & =0 \\
{\left[M_{a b}, P_{c}\right] } & =-i\left(\eta_{a c} P_{b}-\eta_{b c} P_{a}\right) \\
{\left[M_{a b}, M_{c d}\right] } & =-i\left(\eta_{a c} M_{b d}-\eta_{a d} M_{b c}-\eta_{b c} M_{a d}+\eta_{b d} M_{a c}\right) .
\end{aligned}
$$


The spin-matrices $\Sigma_{a b}$ commute with $L_{a b}$ and $P_{a}$, and satisfy the same commutation relations as $M_{a b}(2.7 \mathrm{c})$ - the Lorentz algebra.

Now we consider the local Poincaré (gauge) group. In order to make the Lagrangian $L\left(\phi, \partial_{a} \phi\right)$ invariant under the local Poincaré transformations,

$$
\begin{aligned}
x^{\prime} a & =x^{a}+\omega_{b}^{a}(x) x^{b}+\epsilon^{a}(x), \\
\phi^{\prime}\left(x^{\prime}\right) & =\left(1-\frac{i}{2} \omega^{a b}(x) \Sigma_{a b}\right) \phi(x),
\end{aligned}
$$

with the parameters $\omega^{a b}(x)$ and $\epsilon^{a}(x)$ depending on space-time coordinates, we have to introduce new compensating fields $e^{a}{ }_{\mu}(x)$ and $\omega_{\mu}^{a b}(x)=-\omega_{\mu}^{b a}(x)$, named tetrads and spinconnections, respectively [38]. They enable us to define the gauge covariant derivative [32, 33] (see also [34]), which in the C-frame is written as

$$
\nabla_{\mu} \phi=\left(\partial_{\mu}+\frac{i}{2} \omega_{\mu}^{a b} \Sigma_{a b}\right) \phi .
$$

The gauge fields $e^{a}{ }_{\mu}$ have inverses $e_{a}{ }^{\mu}$ which satisfy

$$
e_{\mu}^{a} e_{b}^{\mu}=\delta_{b}^{a}, \quad e_{\mu}^{a} e_{a}^{\nu}=\delta_{\mu}^{\nu} .
$$

They can be used to transform $\mathrm{C}$-frame indices $\mu, \nu, \ldots$ into the L-frame indices $a, b, \ldots$, and vice versa. Thus we can define the covariant derivative with respect to the L-frame by

$$
\nabla_{a} \phi=e_{a}^{\mu} \nabla_{\mu} \phi
$$

By introducing (2.9) into (2.11), we obtain

$$
\nabla_{a} \phi=i\left(e_{a}^{\mu} P_{\mu}+\frac{1}{2} \omega_{a}^{b c} \Sigma_{b c}\right) \phi .
$$

The expression

$$
\mathcal{A}_{a}=i\left(e_{a}^{\mu} P_{\mu}+\frac{1}{2} \omega_{a}^{b c} \Sigma_{b c}\right)
$$

is also considered as a definition of the gauge potentials valued in the Lie algebra of the Poincaré group [33, 34].

The commutator of two covariant derivatives $\nabla_{a}$ and $\nabla_{b}$ can be calculated by using (2.11) and (2.10):

$$
\left[\nabla_{a}, \nabla_{b}\right] \phi=\left(\frac{1}{2} F_{a b}^{c d} \Sigma_{c d}-F_{a b}^{c} \nabla_{c}\right) \phi
$$

where

$$
F_{a b}^{c d}=F_{\mu \nu}^{c d} e_{a}^{\mu} e_{b}^{\nu}, \quad F_{a b}^{c}=F_{\mu \nu}^{c} e_{a}^{\mu} e_{b}^{\nu},
$$


and

$$
\begin{gathered}
R_{\mu \nu}^{a b} \equiv F_{\mu \nu}^{a b}=\partial_{\mu} \omega_{\nu}^{a b}-\partial_{\nu} \omega_{\mu}^{a b}+\left(\omega_{\mu}{ }^{a c} \omega_{\nu}{ }^{d b}-\omega_{\nu}{ }^{a c} \omega_{\mu}{ }^{d b}\right) \eta_{c d} \\
T_{\mu \nu}^{a} \equiv F_{\mu \nu}^{a}=\partial_{\mu} e^{a}{ }_{\nu}-\partial_{\nu} e^{a}{ }_{\mu}+\left(\omega_{\mu}{ }^{a b} e^{c}{ }_{\nu}-\omega_{\nu}{ }^{a b} e^{c}{ }_{\mu}\right) \eta_{b c} .
\end{gathered}
$$

The quantities $R^{a b}{ }_{\mu \nu}$ and $T_{\mu \nu}^{a}$ are identified with the components of the curvature and torsion tensors of the space-time, respectively. Therefore the Poincaré gauge theory of gravitation has the geometric structure of the Riemann-Cartan space $\mathcal{U}_{4}$ with curvature and torsion.

The metric tensor can be defined by using the tetrad gauge fields. In a C-frame it has the components

$$
g_{\mu \nu}(x)=\eta_{a b} e_{\mu}^{a} e_{\nu}^{b} .
$$

According to (2.18) the metric itself can be seen as an effective gauge field, i.e. a dynamical variable.

By imposing the condition of null torsion, $T_{\mu \nu}^{a}=0$, one can solve for the spin-connection $\omega_{\mu}^{a b}$ in terms of the tetrads $e^{a}{ }_{\mu}$, thus reducing the Einstein-Cartan theory to GR.

\section{Twisting the Poincaré algebra}

Space-time noncommutativity is a way to deform the classical space-time, so that nonlocality becomes its characteristic feature. This means that the notion of a point is no longer welldefined. On such a noncommutative space-time physical phenomena are naturally nonlocal. The coordinates $\hat{x}^{\mu}$ of noncommutative space-time satisfy the commutation relations

$$
\left[\hat{x}^{\mu}, \hat{x}^{\nu}\right]=i \theta^{\mu \nu}
$$

where, in the simplest (canonical) case, $\theta^{\mu \nu}$ is an antisymmetric constant matrix of dimension length-squared. This kind of space-time is described by using the methods of noncommutative geometry. It led to an algebraic description of noncommutative space-times - based entirely on algebraic functions - and it enabled one to define Yang-Mills gauge theories on a large class of noncommutative spaces. For quite some time, the physical applications were based on geometric interpretations of the standard model and its various fields and coupling constants.

To describe physics on the noncommutative space-time generated by (3.1), one replaces the usual point-wise product of functions, $f(x)$ and $g(x)$, by the noncommutative Moyal $\star$-product, which can be written explicitly as

$$
\begin{aligned}
(f \star g)(x) & =f(x) \exp \left(\frac{i}{2} \overleftarrow{\partial}_{\mu} \theta^{\mu \nu} \vec{\partial}_{\nu}\right) g(x) \\
= & f(x) g(x)+\sum_{n=1}^{\infty} \frac{1}{n !}\left(\frac{i}{2}\right)^{n} \theta^{\mu_{1} \nu_{1}} \cdots \theta^{\mu_{n} \nu_{n}}\left(\partial_{\mu_{1}} \cdots \partial_{\mu_{n}} f(x)\right)\left(\partial_{\nu_{1}} \cdots \partial_{\nu_{n}} g(x)\right)
\end{aligned}
$$


Particularly, the commutator of field operators, $\hat{\phi}(x)$ and $\hat{\psi}(x)$, is represented on the algebra of functions by the Moyal bracket:

$$
[\phi(x), \psi(x)]_{\star}=\phi(x) \star \psi(x)-\phi(x) \star \psi(x) .
$$

The defining commutation relations of the noncommutative space-time (3.1) are clearly not covariant under Lorentz transformations, because the left-hand side of the relation is a tensor and the right hand-side is a constant. Thus the noncommutative space-time does not posses Lorentz symmetry. This could be a serious problem, because the quantum and gauge field theories of high energy physics are vitally dependent on the representation content of the Poincaré algebra. The solution to the problems arising from the breaking of the Lorentz symmetry is the twisted Poincaré symmetry [13, 14].

The adequate mathematical framework for describing noncommutative gauge theories and the twisted Poincaré symmetry is that of the Hopf algebras. For Lie algebras one starts with their universal enveloping algebras, which are the most general unital associative algebras into which they can be embedded.

Let us consider a Lie algebra $\mathcal{G}$ generated by $T_{i}, i=1,2, \ldots, n$,

$$
\left[T_{i}, T_{j}\right] \equiv T_{i} T_{j}-T_{j} T_{i}=i f_{i j}^{k} T_{k}
$$

where the associative product is $T_{i} T_{j}$. Its universal enveloping algebra $\mathcal{U}(\mathcal{G})$ consists of the polynomials in the generators $T_{i}$ modulo the commutation relations (3.4), and of the unit element 1. The basis of the universal enveloping algebra can be chosen to consists of 1 and of the fully symmetrized products of the generators

$$
T_{\left(i_{1}\right.} T_{i_{2}} \cdots T_{\left.i_{n}\right)}, n \in \mathbb{N}
$$

The universal enveloping algebra $\mathcal{U}(\mathcal{G})$ can be extended to a Hopf algebra $\mathcal{H}$. The algebra $\mathcal{U}(\mathcal{G})$ consists of a vector space $V$ over the field $\mathbb{C}$ and of the multiplication and unit linear maps

$$
\begin{gathered}
m: V \otimes V \rightarrow V, \\
\eta: \mathbb{C} \rightarrow V,
\end{gathered}
$$

respectively. Explicitly the multiplication is usually written as

$$
m(X \otimes Y)=X Y \quad ; X, Y \in \mathcal{U}(\mathcal{G})
$$

The multiplication $m$ is associative

$$
m \circ(\mathbf{1} \otimes m)=m \circ(m \otimes \mathbf{1})
$$

and the unit map $\eta$ implies the existence of a unit element $\mathbf{1}$ in $V$

$$
m \circ(\mathbf{1} \otimes \eta)=m \circ(\eta \otimes \mathbf{1})=\mathrm{id}(\text { identity map }) .
$$


The bialgebra structure for $\mathcal{U}(\mathcal{G})$ is constructed by introducing the coproduct and counit homomorphisms

$$
\begin{aligned}
& \Delta: V \rightarrow V \otimes V, \\
& \varepsilon: V \rightarrow \mathbb{C},
\end{aligned}
$$

respectively. The coproduct $\Delta$ is coassociative,

$$
(\mathrm{id} \otimes \Delta) \Delta=(\Delta \otimes \mathrm{id}) \Delta
$$

and the counit $\varepsilon$ satisfies

$$
(\mathrm{id} \otimes \varepsilon) \circ \Delta=(\varepsilon \otimes \mathrm{id}) \circ \Delta .
$$

The Hopf algebra is completed by introducing the antipode $S$, an antihomomorphism that is compatible with the bialgebra structure

$$
S: V \rightarrow V, m \circ(S \otimes \mathbf{1}) \circ \Delta=m \circ(\mathbf{1} \otimes S) \circ \Delta=\eta \circ \varepsilon
$$

The Hopf algebra structure of $\mathcal{U}(\mathcal{G})$ is defined by

$$
\begin{aligned}
\Delta_{0}(X) & =X \otimes \mathbf{1}+\mathbf{1} \otimes X, & \Delta_{0}(\mathbf{1}) & =\mathbf{1} \otimes \mathbf{1}, \\
\varepsilon(X) & =0, & \varepsilon(\mathbf{1}) & =1, \\
S(X) & =-X, & S(\mathbf{1}) & =\mathbf{1},
\end{aligned}
$$

for all $X \in V-\{\mathbf{1}\}$. The Hopf algebra $\mathcal{U}(\mathcal{G})$ is noncommutative, but cocommutative due to the symmetry of the coproduct (3.14).

We can deform a cocommutative Hopf algebra like $\mathcal{U}(\mathcal{G})$ to a non-cocommutative one by introducing a twist element

$$
\mathcal{F} \in \mathcal{U}(\mathcal{G}) \otimes \mathcal{U}(\mathcal{G})
$$

and by redefining the coproduct of the Hopf algebra by a similarity transformation

$$
\Delta_{0}(X) \longrightarrow \Delta_{t}(X)=\mathcal{F} \Delta_{0}(X) \mathcal{F}^{-1}, \quad X \in \mathcal{G}
$$

in other words by twisting the coproduct of $\mathcal{U}(\mathcal{G})$ [39] (see also the monographs [40]). In order to preserve the Hopf algebra structure, the twist element has to satisfy the twist conditions

$$
\begin{aligned}
\mathcal{F}_{12}\left(\Delta_{0} \otimes \mathrm{id}\right) \mathcal{F} & =\mathcal{F}_{23}\left(\mathrm{id} \otimes \Delta_{0}\right) \mathcal{F} \\
(\varepsilon \otimes \mathrm{id}) \mathcal{F} & =\mathbf{1}=(\mathrm{id} \otimes \varepsilon) \mathcal{F}
\end{aligned}
$$

where $\mathcal{F}_{12}=\mathcal{F} \otimes \mathbf{1}$ and $\mathcal{F}_{23}=\mathbf{1} \otimes \mathcal{F}$. We denote the twist deformed algebra by $\mathcal{U}_{t}(\mathcal{G})$. The twist element does not affect the multiplication $m$ of the algebra $\mathcal{U}_{t}(\mathcal{G})$ and therefore the commutation relations (3.4) among the generators of $\mathcal{U}(\mathcal{G})$ are preserved. This means that the representation content of $\mathcal{U}_{t}(\mathcal{G})$ is identical with that of $\mathcal{U}(\mathcal{G})$. What is affected by the 
twist, is the action of $\mathcal{U}_{t}(\mathcal{G})$ onto the tensor products of its representations, i.e. the Leibniz rule.

The solution to the problem of representations in noncommutative quantum field theory, due to the non-invariance under Lorentz transformations, was proposed in [13, 14] in the form of the twisted Poincaré symmetry. A twist deformation of the universal enveloping algebra $\mathcal{U}(\mathcal{P})$ of the Poincaré algebra $\mathcal{P}$ was introduced, providing a new symmetry that is respected by the noncommutative theory obtained by Weyl quantization on the noncommutative spacetime (3.1). Since the twist deformation does not alter the multiplication in $\mathcal{U}(\mathcal{P})$, the commutation relations among its generators (2.7) are preserved. Thus the representation content of the twisted algebra $\mathcal{U}_{t}(\mathcal{P})$ is the same as the representation content of the usual Poincaré algebra. This legitimates the usage of the familiar representations of the Poincaré symmetry in the context of noncommutative field theories.

The Poincaré algebra $\mathcal{P}(2.7)$ has a commutative subalgebra of translation generators $P_{\mu}=-i \partial_{\mu}$ that can be used to construct the Abelian twist element

$$
\mathcal{F}=e^{\frac{i}{2} \theta^{\mu \nu} P_{\mu} \otimes P_{\nu}}
$$

where $\theta^{\mu \nu}$ is the real constant antisymmetric matrix in (3.1). It clearly satisfies the twist conditions (3.18)-(3.19) and thus it can be used to consistently twist the coproduct (3.14) of the Hopf algebra $\mathcal{U}(\mathcal{P})$. The coproduct of the translation generators $P_{\mu}$ is not affected by the twist (3.20) due to the commutativity of translations (2.7a),

$$
\Delta_{t}\left(P_{\mu}\right)=\Delta_{0}\left(P_{\mu}\right)=P_{\mu} \otimes \mathbf{1}+\mathbf{1} \otimes P_{\mu} .
$$

The coproduct of the Lorentz generators $M_{\mu \nu}$ is altered by the twist, because of their nonvanishing commutation relations with $P_{\mu}$ (see (2.7b) $)$,

$$
\Delta_{t}\left(M_{\mu \nu}\right)=\Delta_{0}\left(M_{\mu \nu}\right)-\frac{1}{2} \theta^{\rho \sigma}\left(\left(\eta_{\rho \mu} P_{\nu}-\eta_{\rho \nu} P_{\mu}\right) \otimes P_{\sigma}+P_{\rho} \otimes\left(\eta_{\sigma \mu} P_{\nu}-\eta_{\sigma \nu} P_{\mu}\right)\right) .
$$

Since the Abelian twist element (3.20) only involves the generators $P_{\mu}$, only the coordinate dependency of the fields $\phi(x)$ is involved in the deformed multiplication of the fields. Therefore the matrix-valued generators $\Sigma_{\mu \nu}$ act on the component degrees of freedom of the fields $\phi(x)$ in the same way, in the deformed and non-deformed algebra cases, i.e. through the matrix multiplication and the symmetric coproduct

$$
\Delta_{t}\left(\Sigma_{\mu \nu}\right)=\Delta_{0}\left(\Sigma_{\mu \nu}\right)=1 \otimes \Sigma_{\mu \nu}+\Sigma_{\mu \nu} \otimes 1
$$

It should, however, be mentioned that the definition of fields on noncommutative space-time is more involved than in the commutative theory [15, 16].

The noncommutative quantum field theories built through Weyl quantization and the canonical $\star$-product (3.2) possess the twisted Poincaré symmetry, which represents the concept of relativistic invariance in noncommutative field theories. This also enables us to adopt the point of view according to which the noncommutativity of coordinates (3.1) is required by the twisted Poincaré symmetry of space-time. 


\section{Gauging the twisted Poincaré symmetry}

The local Poincaré symmetry is an external gauge symmetry. Through geometrical interpretation the Poincaré gauge symmetry translates to the covariance under general coordinate transformations and to the local Lorentz symmetry. This "duality" of the Poincaré gauge symmetry is both a problem and a possibility, since it has been shown that an internal gauge symmetry can not be twisted together with the Poincaré symmetry [17, 18]. We can attempt to gauge the twisted Poincaré algebra itself and find out whether the gauge theory of the Poincaré symmetry on noncommutative space-time can be formulated by means of a gauge-covariant twist.

We could take the direct naive approach and try to construct a noncommutative gauge theory of the twisted Poincare symmetry by using the Abelian twist (3.20) and by replacing the point-wise product of functions with the Moyal «-product in the classical theory constructed in Section 2. The result would, however, be an inconsistent frame-dependent theory (due to the frame-dependence of the $\star$-product) — in many ways similar to those already developed - that can not be a plausible theory of gravitation. We would not be able to give any meaningful geometrical interpretation to a theory of this type.

Since the global Poincaré symmetry is twisted with the Abelian twist (3.20) in the case of the flat noncommutative space-time, also the generalized Poincaré gauge symmetry on noncommutative space-time should be a quantum symmetry. A natural way to generalize the Poincare gauge symmetry to the noncommutative setting is to consider it as a twisted gauge symmetry, so that the global twisted Poincaré symmetry is obtained in the limit of vanishing gauge fields. When the global twisted Poincaré symmetry is generalized to a gauge symmetry, we have to introduce the gauge fields in order to compensate the non-covariance of the partial derivatives, similarly as in the commutative case. Partial derivatives $\partial_{\mu}$ will be replaced by covariant derivatives, which in the coordinate frame read

$$
\nabla_{\mu}=d_{\mu}+\mathcal{A}_{\mu}(x)=i\left(e^{a}{ }_{\mu}(x) P_{a}+\frac{1}{2} \omega_{\mu}^{a b}(x) \Sigma_{a b}\right),
$$

where the $\Sigma_{\nu \rho}$ generate a finite-dimensional representation of the Lorentz algebra. The difference compared to the covariant derivative of an internal gauge symmetry [18]

$$
D_{\mu}=\partial_{\mu}+i A_{\mu}(x)=i\left(P_{\mu}+A_{\mu}^{a}(x) T_{a}\right)
$$

are the tetrad gauge fields $e^{a}{ }_{\mu}$ multiplying $P_{\mu}$ in (4.1). $\mathcal{A}_{\mu}$ are the gauge fields associated to the local Lorentz transformations. In order to obtain a theory that is covariantly deformed under the Poincaré gauge transformations, the frame-dependent translation generators $P_{\mu}$ have to be replaced by the covariant derivatives $-i \nabla_{\mu}$ in the Abelian twist element (3.20). The covariant non-Abelian twist element is of the form

$$
\mathcal{T}=e^{-\frac{i}{2} \theta^{\mu \nu} \nabla_{\mu} \otimes \nabla_{\nu}+\mathcal{O}\left(\theta^{2}\right)},
$$


where $\mathcal{O}\left(\theta^{2}\right)$ stands for the possible additional covariant terms in higher orders of the noncommutativity parameter $\theta^{\mu \cdot 1}$. Because of the similar forms of the covariant derivatives (4.1) and (4.2) and of their twist elements, the basic algebraic reasoning presented in [18] holds also for the twist element (4.3) proposed here. The gauge fields $\mathcal{A}_{\mu}$ alone in $\nabla_{\mu}$ will violate the twist condition (3.18) and the rest of gauge fields $e^{a}{ }_{\mu}$ are not able to rescue the twist condition. The fact that there are now two second rank (field strength) tensors (2.16)-(2.17) does not help to satisfy the twist condition.

Following the arguments of [18], we can attempt to impose the twist condition (3.18). First we consider the twist element (4.3) with only the first order term in $\theta$ in the exponent. The second order terms in $\theta$ that do not cancel in the twist condition (3.18) are, in the left-hand side

$$
\begin{aligned}
& \frac{1}{2}\left(-\frac{i}{2}\right)^{2} \theta^{\mu \nu} \theta^{\rho \sigma}\left(2 \nabla_{\mu} \nabla_{\rho} \otimes \nabla_{\nu} \otimes \nabla_{\sigma}+2 \nabla_{\mu} \otimes \nabla_{\nu} \nabla_{\rho} \otimes \nabla_{\sigma}\right. \\
&\left.+\nabla_{\mu} \otimes \nabla_{\rho} \otimes \nabla_{\nu} \nabla_{\sigma}+\nabla_{\rho} \otimes \nabla_{\mu} \otimes \nabla_{\nu} \nabla_{\sigma}\right)
\end{aligned}
$$

and in the right-hand side

$$
\begin{aligned}
& \frac{1}{2}\left(-\frac{i}{2}\right)^{2} \theta^{\mu \nu} \theta^{\rho \sigma}\left(2 \nabla_{\rho} \otimes \nabla_{\mu} \nabla_{\sigma} \otimes \nabla_{\nu}+2 \nabla_{\rho} \otimes \nabla_{\mu} \otimes \nabla_{\nu} \nabla_{\sigma}\right. \\
&\left.+\nabla_{\mu} \nabla_{\rho} \otimes \nabla_{\nu} \otimes \nabla_{\sigma}+\nabla_{\mu} \nabla_{\rho} \otimes \nabla_{\sigma} \otimes \nabla_{\nu}\right) .
\end{aligned}
$$

These terms can not be canceled by terms that contain second rank tensors

$$
R_{\mu \nu}^{a b} \Sigma_{a b}, \quad T_{\mu \nu}^{a} \nabla_{a}
$$

because the two indices for such tensors come from the same $\theta^{\mu \nu}$, unlike for the $\nabla \nabla$ factors in (4.4) and (4.5). This is why such terms are not included in twist element (4.3) in the first place. The other possible second order terms in (4.3) have the forms

$$
\begin{array}{lr}
\theta^{\mu \nu} \theta^{\rho \sigma} 1 \otimes \nabla_{\mu} \nabla_{\nu} \nabla_{\rho} \nabla_{\sigma}, & \theta^{\mu \nu} \theta^{\rho \sigma} \nabla_{\mu} \nabla_{\nu} \nabla_{\rho} \nabla_{\sigma} \otimes 1, \\
\theta^{\mu \nu} \theta^{\rho \sigma} \nabla_{\mu} \otimes \nabla_{\nu} \nabla_{\rho} \nabla_{\sigma}, & \theta^{\mu \nu} \theta^{\rho \sigma} \nabla_{\mu} \nabla_{\nu} \nabla_{\rho} \otimes \nabla_{\sigma}, \\
\theta^{\mu \nu} \theta^{\rho \sigma} \nabla_{\mu} \nabla_{\nu} \otimes \nabla_{\rho} \nabla_{\sigma}, &
\end{array}
$$

with all the permutations of indices of the covariant derivatives - although the antisymmetry of $\theta$ greatly reduces the number of independent permutations. We have verified that when introduced into the twist element (4.3) and consequently into the twist condition (3.18), these second orders terms can never cancel all the terms in (4.4) and (4.5). Therefore, the twist condition (3.18) can not be fulfilled in the second order in $\theta$.

It is well known that the gauging of the translation symmetry leads to the EinsteinHilbert Lagrangian and to the covariance under general coordinate transformations [34].

\footnotetext{
${ }^{1}$ The following discussion is presented for the exponential form (4.3), but the results are valid for any invertible functional form.
} 
Hence, it is interresting to see whether the gauge theory of the external translation symmetry group $\mathcal{T}_{4}$ can be consistently defined together with the twisted Poincaré symmetry. The covariant derivative for the local translations is

$$
d_{\mu}=i e_{\mu}^{a} P_{a}
$$

In fact, this is also the covariant derivative of the Poincaré gauge symmetry for one-dimensional representations, for which the covariant derivative (4.1) should reduce to (4.10). Clearly the gauge fields $e^{a}{ }_{\mu}$ now contain contributions also from the local Lorentz transformations. Since the covariant derivatives of the translation group do not commute,

$$
\left[d_{\mu}, d_{\nu}\right]=C_{\mu \nu}^{\rho} d_{\rho}, \quad C_{\mu \nu}^{\rho}=\left(e^{a}{ }_{\mu} \partial_{a} e^{b}{ }_{\nu}-e^{a}{ }_{\nu} \partial_{a} e^{b}{ }_{\mu}\right) e_{b}^{\rho}
$$

the covariant element

$$
\mathcal{T}=e^{-\frac{i}{2} \theta^{\mu \nu} d_{\mu} \otimes d_{\nu}+\mathcal{O}\left(\theta^{2}\right)}=e^{\frac{i}{2} \theta^{\mu \nu} e^{a}{ }_{\mu} P_{a} \otimes e^{b}{ }_{\nu} P_{b}+\mathcal{O}\left(\theta^{2}\right)}
$$

can not be of the Abelian type (3.20), which is known to be a twist. Because of this and the high level of arbitrariness in choosing the gauge fields $e^{a}{ }_{\mu}$ in the translationally covariant twist (4.12), we face similar algebraic problems as with the covariant twist element (4.3) of the full Poincaré gauge symmetry. The twist element (4.12) does not satisfy the twist condition (3.18), even though its form is much simpler now. Thus, it is not only the local Lorentz symmetry that breaks the validity of the non-Abelian Poincaré gauge covariant twist element (4.3); the external gauge symmetry associated with the general coordinate transformations is just as problematic.

Thus, we have obtained the result that the Poincaré gauge covariant non-Abelian element (4.3) is not a twist and the $\star$-product defined by it is not associative. We can conclude that the twisted Poincaré symmetry can not be gauged by generalizing the Abelian twist (3.20) to a covariant non-Abelian twist (4.3), nor by introducing a more general covariant twist element.

It should be mentioned that from the mathematical point of view, we could try to deform the action of the twisted Poincaré algebra on its representations, instead of generalizing the twist element, but it seems unlikely that such an approach could solve the problems related to the frame-dependent twist element (3.20).

\section{Concluding remarks and perspectives}

In this paper we have investigated the possibility of gauging the twisted Poincaré symmetry in order to obtain a noncommutative gauge theory of gravitation. A covariant non-Abelian twist element $\mathcal{T}$ has been defined by using the covariant derivative of the commutative Poincaré gauge theory. The twist condition that assures the associativity of the multiplication on the representations of the twisted Poincaré algebra is violated already in the second order 
in the noncommutativity parameter $\theta^{\mu \nu}$. Adding gauge covariant terms of higher orders in $\theta^{\mu \nu}$ into the definition of the twist $\mathcal{T}$ does not improve the result. When we restrict the gauge symmetry to the translation group $\mathcal{T}_{4}$, we are faced with similar algebraic problems as in the case of the full Poincaré symmetry. Thus both the local Lorentz symmetry and the local translational symmetry, associated with the covariance under general coordinate transformations, violate the twist condition already in the second order in the parameter $\theta^{\mu \nu}$.

The question of unifying the external (global or local) Poincaré symmetry and the internal gauge symmetry under a common twist remains an open fundamental problem of noncommutative gauge theories.

Since the introduction of a gauge covariant twist breaks the associativity of the algebra of functions on noncommutative space-time, both in the internal and external gauge symmetry cases, we may have to consider space-time geometries that are also nonassociative, not only noncommutative. Indeed, there exist in the literature works on constructing nonassociative theories with some desired properties (see, e.g., 41, 42, 43, and references therein).

The nonassociativity, as well as the noncommutativity, has its origin in string theory. It is known that in the presence of a constant background field, $\omega=B+F$, the noncommutative geometry is described by the Moyal product which is associative [4] (see also [44, 45]). The physics of this case, corresponding to a flat brane embedded in a flat background space, is well understood [46]. When $\omega$ is not constant, but it satisfies $\mathrm{d} \omega=0$, the target space becomes a Poisson manifold and thus the Kontsevich prescription [47] can be used to define the associative product. In the most general case, $\mathrm{d} \omega \neq 0$, it has been established that the extension of the Kontsevich as well as the Moyal products become nonassociative [44].

Defining gauge theory on nonassociative manifolds is not straightforward. Recently, there have been attempts to restore the associativity of the star-product, when the ordinary derivative is replaced by the covariant derivative. In [48] (see also [25] for details and example) was considered a curved background and a $\theta^{\mu \nu}$ which is a covariantly constant antisymmetric tensor, $D_{\mu} \theta^{\nu \rho}=0$. However, the appearance of the commutators $\left[D_{\mu}, D_{\nu}\right]$ in the star-product, which vanish only on scalar functions, spoils the associativity. By considering a background space endowed with a Friedmann-Robertson-Walker metric, it was found [48] that $\theta^{\mu \nu}$ can be chosen such that the nonassociativity appears at the fourth order in $\theta$, while the noncommutative effects are already present starting with $\theta^{2}$. No extension to a "complete" associative star-product has, however, been obtained.

In [45] it was suggested that such a nonassocitivity "anomaly" can be removed by including the Chan-Paton factors to define the associative star-product, starting from the axioms of the rational conformal field theory. It is argued that by using the vacuum string field theory, one may push most of the D-branes in a so-called "closed string vacuum". In this case the associativity is restored, i.e. the Chan-Paton factors modify the originally nonassociative algebra to an associative one. An infinite number of D-branes are, however, needed for this modification. 
In the gauge theory of the twisted Poincaré algebra proposed in our work, the twist condition (3.18) is not satisfied. This means that the algebra of the twist symmetry does not close, a property which also implies the nonassociativity of the star-product.

We believe, however, that in formulating the gauge theory of noncommutative gravity, the requirement of general coordinate transformations with respect to the whole Lorentz group should be relaxed and replaced by the requirement of general coordinate transformations only under the residual symmetry of the noncommutative field theories as argued in [16]. This approach will be pursued in a forthcoming communication [49. In a quite different context, the description of Nature at the Planck scale is suggested to be given by a nonlocal translationally invariant theory, the so-called "Very Special Relativity", with a symmetry under a subgroup of the Lorentz group [50], while at low-energy scale the Poincaré invariance would be operating. A realization of such a symmetry, for the Planck scale part, has been recently given [51] on the noncommutative space-time with light-like noncommutativity. A gauge theory of the latter symmetry can be performed as mentioned above [49].

\section{Acknowledgements}

We are indebted to Kazuhiko Nishijima, Ruibin Zhang and Xiao Zhang for discussions during different stages of this work. A. T. acknowledges the project no. 121720 of the Academy of Finland. G. Z. acknowledges the support by the CNCSIS-UEFISCSU grant 620 of the Minister of Education, Research and Youth of Romania.

\section{References}

[1] H. S. Snyder, Quantized Space-Time, Phys. Rev. 71 (1947) 38.

[2] S. Doplicher, K. Fredenhagen and J. E. Roberts, Spacetime quantization induced by classical gravity, Phys. Rev. B 331 (1994) 33.

[3] S. Doplicher, K. Fredenhagen and J. E. Roberts, The quantum structure of spacetime at the Planck scale and quantum fields, Commun. Math. Phys 172 (1995) 18, arXiv:hep-th/0303037.

[4] N. Seiberg and E. Witten, String theory and noncommutative geometry, JHEP 09 (1999) 032, arXiv:hep-th/9908142.

[5] M. Chaichian, P. Prešnajder, M. M. Sheikh-Jabbari and A. Tureanu, Noncommutative gauge field theories: a no-go theorem, Phys. Lett. B 526 (2002) 132, arXiv:hep-th/0107037.

[6] S. Terashima, A note on superfields and noncommutative geometry, Phys. Lett. B 482 (2000) 276, arXiv:hep-th/0002119. 
[7] J. M. Gracia-Bondia and C. P. Martin, Chiral gauge anomalies on noncommutative $\mathbf{R}^{4}$, Phys. Lett. B 479 (2000) 321, arXiv:hep-th/0002171.

[8] M. Chaichian, P. Prešnajder, M. M. Sheikh-Jabbari and A. Tureanu, Noncommutative standard model: Model building, Eur. Phys. J. C 29 (2003) 413, arXiv:hep-th/0107055; M. Chaichian, A. Kobakhidze and A. Tureanu, Spontaneous reduction of noncommutative gauge symmetry and model building, Eur. Phys. J. C 47 (2006) 241, arXiv:hep-th/0408065.

[9] M. Arai, S. Saxell and A. Tureanu, A Noncommutative version of the minimal supersymmetric standard model, Eur. Phys. J. C 51 (2007) 217, arXiv: hep-th/0609198.

[10] J. Madore, S. Schraml, P. Schupp and J. Wess, Gauge theory on noncommutative spaces, Eur. Phys. J. C 16 (2000) 161, arXiv:hep-th/0001203.

[11] B. Jurčo, S. Schraml, P. Schupp and J. Wess, Enveloping algebra-valued gauge transformations for non-abelian gauge groups on non-commutative spaces, Eur. Phys. J. C 17 (2000) 521, arXiv:hep-th/0006246.

[12] X. Calmet, B. Jurčo, P. Schupp, J. Wess and M. Wohlgenannt, The standard model on non-commutative space-time, Eur. Phys. J. C 23 (2002) 363, arXiv:hep-ph/0111115.

[13] M. Chaichian, P. Kulish, K. Nishijima and A. Tureanu, On a Lorentz-invariant interpretation of noncommutative space-time and its implications on noncommutative QFT, Phys. Lett. B 604 (2004) 98, arXiv:hep-th/0408069.

[14] M. Chaichian, P. Prešnajder and A. Tureanu, New concept of relativistic invariance in NC space-time: Twisted Poincaré symmetry and its implications, Phys. Rev. Lett. 94 (2005) 151602, arXiv:hep-th/0409096.

[15] M. Chaichian, P. P. Kulish, A. Tureanu, R. B. Zhang and X. Zhang, Noncommutative fields and actions of twisted Poincaré algebra, J. Math. Phys. 49 (2008) 042302, arXiv:0711.0371 [hep-th].

[16] M. Chaichian, K. Nishijima, T. Salminen and A. Tureanu, Noncommutative Quantum Field Theory: A Confrontation of Symmetries, JHEP 06 (2008) 078, arXiv:0805.3500 [hep-th].

[17] M. Chaichian and A. Tureanu, Twist symmetry and gauge invariance, Phys. Lett. B 637 (2006) 199, arXiv:hep-th/0604025.

[18] M. Chaichian, A. Tureanu and G. Zet, Twist as a Symmetry Principle and the Noncommutative Gauge Theory Formulation, Phys. Lett. B 651 (2007) 319, arXiv:hep-th/0607179. 
[19] S. Coleman and J. Mandula, All Possible Symmetries of the S-Matrix, Phys. Rev. 159 (1967) 1251.

[20] R. Haag, J. T. Lopuszansky and M. Sohnius, All possible generators of supersymmetries of the S-matrix, Nucl. Phys. B 88 (1975) 257.

[21] A. H. Chamseddine, Complexified gravity in noncommutative spaces, Commun. Math. Phys. 218 (2001) 283, arXiv:hep-th/0005222.

[22] A. H. Chamseddine, Deforming Einstein's gravity, Phys. Lett. B 504 (2001) 33, arXiv:hep-th/0009153.

[23] A. H. Chamseddine, Invariant actions for noncommutative gravity, J. Math. Phys. 44 (2003) 2534, arXiv:hep-th/0202137.

[24] P. Aschieri, C. Blohmann, M. Dimitrijevic, F. Meyer, P. Schupp and J. Wess, A gravity theory on noncommutative spaces, Class. Quant. Grav. 22 (2005) 3511, arXiv:hep-th/0504183.

[25] E. Harikumar and V. O. Rivelles, Noncommutative gravity, Class. Quant. Grav. 23 (2006) 7551, arXiv:hep-th/0607115.

[26] M. Chaichian, A. Tureanu and G. Zet, Corrections to Schwarzschild solution in noncommutative gauge theory of gravity, Phys. Lett. B 660 (2008) 573, arXiv:0710.2075 [hep-th];

M. Chaichian, A. Tureanu, M. R. Setare and G. Zet, On Black Holes and Cosmological Constant in Noncommutative Gauge Theory of Gravity, JHEP 04 (2008) 064, arXiv:0711.4546 [hep-th].

[27] H. Steinacker, Emergent Gravity from Noncommutative Gauge Theory, JHEP 12 (2007) 049, arXiv:0708.2426 [hep-th].

[28] H. Grosse, H. Steinacker and M. Wohlgenannt, Emergent Gravity, Matrix Models and UV/IR Mixing, JHEP 04 (2008) 023, arXiv:0802.0973 [hep-th].

[29] X. Calmet and A. Kobakhidze, Noncommutative general relativity, Phys. Rev. D 72 (2005) 045010, arXiv:hep-th/0506157.

[30] L. Álvarez-Gaumé, F. Meyer and M. A. Vázquez-Mozo, Comments on noncommutative gravity, Nucl. Phys. B 753 (2006) 92, arXiv:hep-th/0605113.

[31] M. Chaichian, A. Tureanu, R. Zhang and Xiao Zhang, Riemannian Geometry of Noncommutative Surfaces, J. Math. Phys. (in print), arXiv:hep-th/0612128.

[32] R. Utiyama, Invariant Theoretical Interpretion of Interaction, Phys. Rev. 101 (1956) 1597. 
[33] T. W. B. Kibble, Lorentz Invariance and the Gravitational Field, J. Math. Phys. 2 (1961) 212.

[34] M. Blogojević, Gravitation and gauge symmetries, Institute of Physics Publishing (2001).

[35] D. W. Sciama, The Physical Structure of General Relativity, Rev. Mod. Phys. 36 (1964) 463.

[36] K. Hayashi and A. Bregman, Poincaré gauge invariance and the dynamical role of spin in gravitational theory, Annals Phys. 75 (1973) 562.

[37] F. W. Hehl, P. von der Heyde and G. D. Kerlick, General relativity with spin and torsion, Rev. Mod. Phys. 48 (1976) 393.

[38] Y. M. Cho, Gauge theory of Poincaré symmetry, Phys. Rev. D 14 (1976) 3335.

[39] V. G. Drinfeld, Leningrad Math. J. 1 (1990) 321.

[40] V. Chari and A. Pressley, A Guide to Quantum Groups, Cambridge University Press, Cambridge, 1994;

S. Majid, Foundations of Quantum Group Theory, Cambridge University Press, Cambridge, 1995;

M. Chaichian and A. Demichev, Introduction to Quantum Groups, World Scientific, Singapore, 1996.

[41] A. I. Nesterov and L. V. Sabinin, Nonassociative geometry: FriedmannRobertson-Walker spacetime, Int. J. Geom. Meth. Mod. Phys. 3 (2006) 1481, arXiv:hep-th/0406229.

[42] S. Majid, Gauge theory on nonassociative spaces, J. Math. Phys. 46 (2005) 103519, arXiv:math/0506453.

[43] Y. Sasai and N. Sasakura, One-loop unitarity of scalar field theories on Poincaré invariant commutative nonassociative spacetimes, JHEP 09 (2006) 046, arXiv:hep-th/0604194.

[44] L. Cornalba and R. Schiappa, Nonassociative star-product deformations for Dbrane worldvolumes in curved backgrounds, Commun. Math. Phys 225 (2002) 33, arXiv:hep-th/0101219.

[45] Y. Matsuo, Comments on Resolution of Nonassociativity in SFT - An Example from Axioms of BCFT, Braz. J. Phys. 32 (2002) 861; Projection operators and Dbranes in purely cubic open string field theory, Mod. Phys. Lett. A 16 (2001) 1811, arXiv:hep-th/0107007. 
[46] V. Schomerus, D-brane and deformation quantization, JHEP 06 (1999) 030, arXiv:hep-th/9903205.

[47] M. Kontsevich, Deformation quantization of Poisson manifolds, Lett. Math. Phys. 66 (2003) 157, arXiv:q-alg/9709040.

[48] F. Lizzi, G. Mangano, G. Miele and M. Peloso, Cosmological perturbations and short distance physics from noncommutative geometry, JHEP 06 (2002) 049, arXiv:hep-th/0203099.

[49] Work in progress.

[50] A. G. Cohen and S. L. Glashow, Very Special Relativity, Phys. Rev. Lett. 97 (2006) 021601, arXiv:hep-ph/0601236.

[51] M. M. Sheikh-Jabbari and A. Tureanu, A Realization of Cohen-Glashow Very Special Relativity, arXiv:0806.3699 [hep-th]. 